\title{
La carte du sang de l'immobilier chinois, un cas de cyber- activisme
}

Nicolas DOUAY, maître de conférences en urbanisme, Université Paris-Diderot (PRES Sorbonne Paris Cité), UMR Géographie-Cités, nicolas.douay@gmail.com

Marta SEVERO, ingénieure de recherche, Université de Paris 1, GIS CIST, marta.severo@gis-cist.fr

Timothée GIRAUD, ingénieur d'étude, CNRS, UMS RIATE, timothee.giraud@umsriate.fr

Pre-print. Publié dans Information géographique, Vol.6, n.1, 2012.

RESUME

L'objectif de cet article est d'explorer les nouvelles formes de mobilisation sociale en milieu urbain qui utilisent les technologies de l'Internet. Le développement des réseaux sociaux en ligne offre en effet de nouvelles possibilités d'expression et de contestation. Le Web 2.0 se transforme ainsi en un espace public numérique complémentaire de l'espace public physique traditionnel surtout lorsque celui-ci est particulièrement contrôlé.

Le cas de la "carte du sang de l'immobilier chinois " est particulièrement représentatif de ces transformations. Mise en ligne en octobre 2010, cette carte coopérative fait appel à la connaissance des internautes pour répertorier les développements immobiliers ayant donné lieu à des violences physiques. Celles-ci peuvent aller de la simple répression de manifestations à des immolations par le feu. La version vérifiée de la carte présente 85 événements et la version ouverte 199 cas. La publication de cette carte a été reprise dans les médias chinois et internationaux, participant ainsi à l'inscription sur l'agenda politique international des enjeux sociaux liés au développement urbain et immobilier chinois.

MOTS CLES

Réseaux sociaux, Web 2.0, blog, conflits, immobilier, luttes urbaines, activisme

\section{ABSTRACT}

The aim of this paper is to explore new forms of social mobilization in urban areas that use the Internet technologies. The development of online social networks offers new possibilities of expression and protest. Web 2.0 is transformed into a digital public space complementary to the traditional physical public space especially when this one is particularly controlled.

The Chinese bloody map of real estate is particularly 
representative of these changes. Published in October 2010, this map uses cooperative knowledge of Internet users to list real estate developments that led to physical violence. These can range from simple protest repression to self-immolation. The checked version of the map shows 85 events and the open version 199 cases. The diffusion of the news of this map in the Chinese and international medias helped to put this social issue on the international political agenda related to urban development and real estate in China.

\section{KEYS-WORDS}

Social networks, Web 2.0, blog, conflicts, real estate, urban struggles, activism

\section{Introduction}

L'urbanisation rapide de la Chine se traduit par une multiplication de projets d'infrastructures et d'immobilier. Ce processus de construction s'accompagne de nombreuses destructions, toutefois la modernisation des villes chinoises ne profite pas à tous et laisse apparaître un accroissement des inégalités et de nombreuses luttes urbaines. Alors que l'espace public physique est fortement contrôlé par les autorités, le développement croissant de l'Internet offre de nouvelles possibilités de mobilisation et de contestation pour les activistes urbains qui deviennent de ce fait des cyber-activistes. En déjouant les mécanismes de la censure, les internautes chinois transforment les territoires numériques du Web $2.0^{1}$ en de nouveaux outils de débat public.

À travers l'exemple de «la carte du sang de l'immobilier chinois », l'objectif de cet article est d'explorer les nouvelles formes de mobilisation sociale en milieu urbain et d'interroger l'émergence de nouvelles formes d'activisme urbain en lien avec les technologies de l'information et de la communication.

La collecte des données de «la carte du sang " a été menée en mai 2011 lors de la réalisation d'un mémoire de master (Li \& Wang, 2011), puis l'analyse a été effectuée en croisant deux perspectives théoriques différentes mais complémentaires. D'une part, les théories et les méthodes de l'urbanisme ont permis d'étudier les conflits, les interactions entre les acteurs et finalement les manières d'envisager la production de l'espace urbain chinois. D'autre part, le champ de l'étude des médias, avec l'emploi de nouvelles méthodes numériques d'exploration des données disponibles sur la Toile, a mis en évidence les dynamiques propres à ce cas de cyber-activisme. Deux conférences (Douay N., Severo M. \& Giraud T., 2011 ; Severo M., Giraud T. \& Douay N., 2011) ont permis de présenter des résultats intermédiaires. La première partie de l'article présente le contexte du développement de ces formes de militantisme, la deuxième partie analyse le cas de «la carte du sang de l'immobilier» et enfin la dernière partie met en perspective les processus de production de l'espace urbain, notamment quant à l'apport des nouvelles technologies.

\footnotetext{
${ }^{1}$ Concept proposé par Tim O'Reilly en 2005. Ce concept insiste sur le passage à un web participatif. Internet correspond alors à une intelligence collective où chaque internaute fait partie d'un réseau social. Plus clairement, le Web 2.0 mettrait en scène l'internaute en lui donnant la possibilité d'interagir et de s'identifier à une communauté.
} 


\section{Des luttes urbaines au Web 2.0}

\section{L'urbanisation et le problème des démolitions violentes}

Depuis la politique d'ouverture établie en 1978 par Deng Xiaoping, l'urbanisation et l'économie de la Chine se sont développées rapidement. Par l'intermédiaire d'un exode rural important, le taux d'urbanisation est passé de $20 \%$ en 1979 à $50 \%$ aujourd'hui. Le développement urbain se poursuit, ainsi 350 millions de nouveaux citadins sont attendus d'ici 2025. En 2030, les urbains seront plus d'1 milliard et 221 villes seront millionnaires. Cette transition urbaine se traduit par de nombreux chantiers visant à moderniser le tissu urbain et surtout à l'étendre. À l'horizon 2030, le gouvernement chinois prévoit de réaliser 5 milliards de $\mathrm{m}^{2}$ de routes et 5 millions de bâtiments représentant 40 milliards de $\mathrm{m}^{2}$. Cette production de logements s'accompagne d'une hausse constante des prix et d'importants mouvements spéculatifs, particulièrement dans les grandes métropoles.

Les habitants des quartiers anciens sont, de ce fait, souvent victimes de ces processus spéculatifs en étant relégués de manière plus ou moins violentes en dehors des centres urbains. Malheureusement, peu de données statistiques documentent ce type d'abus. Selon le Centre on Housing Rights and Evictions ${ }^{2}$ (2008, p. 6-7), plus de 40 millions d'agriculteurs chinois ont perdu leurs terres et leurs moyens de subsistance dans les vingt dernières années en raison de l'industrialisation et de l'urbanisation rapides. Au moins 1,25 million de logements ont été démolis et près de 3,7 millions de personnes ont été expulsées et déplacées à travers la Chine dans la dernière décennie, causant beaucoup de souffrances à des milliers de communautés à travers le pays. Le manque de recours juridiques, les compensations et les relogements insuffisants, l'utilisation de la force dans l'exécution des expulsions, ainsi que la brutalité policière envers ceux qui protestent contre l'accaparement des terres et les expulsions forcées sont un témoignage du mépris du gouvernement chinois pour les droits au logement. COHRE rapporte aussi les cas d'expulsions forcées d'au moins 400000 personnes à Pékin en lien avec les Jeux olympiques de 2008 - un chiffre reconnu par l'administration municipale de Pékin. Ainsi, les conflits liés à l'urbanisation rapide et à la spéculation immobilière suscitent la mobilisation des pétitionnaires. Par exemple, en $2003,70 \%$ des demandes concernent les évictions forcées (Human Right Watch, 2003, p. 22).

De plus, les lois qui régulent la démolition des domiciles et l'indemnisation des propriétaires sont assez insuffisantes. En 2001, une première réglementation sur cet enjeu établit l'intérêt national au-dessus des droits individuels et place les promoteurs immobiliers dans une situation privilégiée. Ensuite, en 2007, le vote de la loi sur la propriété relance le débat avec une situation en partie contradictoire. D'une part dans son article 66, la loi donne la priorité aux intérêts des citoyens qui doivent pouvoir jouir en tout temps de leurs propriétés face aux autres particuliers et institutions. Et d'autre part, dans son article 42, elle ouvre de nombreuses possibilités d'expropriations au nom de l'intérêt général. La contradiction entre les différents textes crée un important débat qui sera alimenté par de nombreux professeurs de droit et des activistes engagés dans la défense des droits. Finalement, à la suite de différents scandales d'évictions violentes (notamment la mort de Tang Fuzhen en 2009 qui sera présentée dans la suite de l'article), une nouvelle loi est votée le 21 janvier 2011. Celle-ci permet de préciser la responsabilité des différentes parties, les modalités de la destruction ou encore l'évaluation de la compensation afin d'éviter les conflits les plus violents qui peuvent être

${ }^{2} \mathrm{ONG}$ internationale basée à Genève. 
une source de désordre social pour le gouvernement. Cette avancée juridique peut-être mise en lien avec l'effet direct du débat public suscité par la médiatisation des situations les plus choquantes.

\section{Le développement de l'Internet en Chine}

Le nombre d'internautes chinois connaît une croissance rapide, il a atteint les 500 millions en septembre 2011, ce qui représente un taux de pénétration de près de $40 \%$ de la population mais beaucoup plus dans les grandes villes. Les différents réseaux sociaux dominants dans le monde occidental tels que Youtube, Twitter, Flickr et Facebook sont interdits mais ont leurs équivalents chinois tels que Youku, Renren, $Q Q$ ou Baidu. Ces réseaux sociaux sont utilisés par plus de la moitié des internautes, ainsi les utilisateurs de microblogs sont au nombre de 300 millions. Le gouvernement tente de garder le contrôle sur la Toile avec un important dispositif de censure. La partie pare-feu de ce système est connue à l'extérieur de la Chine continentale sous le nom de "grande muraille pare-feu de Chine » en double référence à son rôle de pare-feu réseau et à la grande muraille. Ainsi certaines expressions, tabous ou sensibles comme "Tibet », sont bloquées sur les moteurs de recherche qu'ils soient chinois ou étrangers. Le web chinois est par conséquent expurgé de sites politiquement sensibles.

Le contrôle du web vise aussi à orienter les échanges entre internautes en évitant que les rumeurs se déploient et que la dissidence se développe. Environ 280000 honkers ou «hacker rouge » sont chargés d'orienter les débats dans les forums et, le cas échéant, de dénoncer les internautes s'éloignant trop de la ligne officielle. Les internautes chinois jonglent toutefois avec la censure en ayant recours au Proxy et au VPN. Ils jouent souvent avec les sous-entendus, et investissent la blogosphère et autres réseaux sociaux de l'ère du Web 2.0 qui constituent des forums inédits d'expression publique et de contestation (Haski P., 2008 ; Yang G., 2009 ; Tai Z., 2006 ; Hermès n '55, 2010 ; Arsène S., 2008 ; Douay N., 2011 ; Severo et al., 2011 ; Kurt Herold \& Marolt, 2011). Ainsi, assez régulièrement, différentes histoires font le buzz et obligent les autorités à réagir soit officiellement, soit par l'intermédiaire de ses honkers.

\section{2. «La carte du sang de l'immobilier»}

\section{La collaboration des internautes, moteur du blog}

En octobre 2010, un internaute anonyme utilisant le pseudonyme de Xuefangditu, soit " carte du sang de l'immobilier », a choisi de répertorier les expulsions violentes liées à des projets immobiliers du pays ${ }^{3}$. Le blogueur veut ainsi dépasser l'émotion que peuvent susciter les violences liées à l'immobilier et en appeler à la responsabilité des citoyens. II veut dénoncer la rapidité du processus d'urbanisation qui détruit la vie de nombreux citoyens et créer un mouvement de boycott de ces nouveaux développements immobiliers où le sang a coulé, perspective qui peut rappeler les traditionnels appels à la désobéissance civile :

«ARTE Journal : Quelle était votre intention en créant cette carte ?

Bloody Map : Mon but est de collecter et de lister les cas d'expulsions

\footnotetext{
${ }^{3}$ Les informations présentées dans cette partie proviennent d'une revue de presse chinoise et internationale, du suivi du blog et du compte Twitter (celui-ci contourne les règles de la censure), ainsi que d'une interview et de différents messages échangés au printemps et à l'été 2011.
} 
violentes qui ont ou auront bientôt disparu du champ de vision du public. II y a des évictions forcées qui se déroulent en ce moment même et qui nécessitent une plus grande couverture médiatique. Pour autant, les répercussions de la "carte des maisons de sang" sont limitées. Les gens ne peuvent pas attendre d'une initiative comme celle-ci qu'elle suscite suffisamment d'attention pour mettre fin aux expulsions forcées actuelles. Le but de cette carte est plutôt de présenter des preuves, pour permettre aux consommateurs de prendre leurs décisions.

ARTE Journal : avec cette carte, vous appelez la population à agir... Bloody Map : II y a 30 ans, la plupart des Chinois pensaient que les intérêts de l'État étaient supérieurs aux intérêts personnels. Mais aujourd'hui, même si la phrase "les biens personnels sont intouchables" est inscrite dans la constitution chinoise, je ne crois pas qu'elle soit inscrite dans le cœur des Chinois. Par exemple, l'immolation de Yihuang, en septembre, a été très suivie par le public. Les médias ont critiqué les responsables du gouvernement local, qui ont finalement été obligés de démissionner. Mais l'avis des habitants locaux était plus nuancé, car ils trouvaient que cet incident empêchait le développement de la région. Alors il faut se poser la question suivante : si il y avait quelqu'un qui refusait de se faire expulser et que ce refus mettait en cause mon intérêt personnel, est ce que je suis tout de même de son côté? Est-ce que je le soutiendrais ? ${ }^{4}$

Le blog utilise l'outil cartographique de Google Map, et sur le modèle de Wikipedia, appelle à la participation des internautes pour collecter, mettre en ligne, vérifier et discuter les informations. À l'été 2011, la version ouverte (voir Carte 1) compte 199 cas rapportés par les internautes et la version vérifiée (voir Carte 2) rassemble uniquement 85 cas qui ont été validés par le bloggeur ${ }^{5}$. Pour rapporter un cas, chaque internaute doit fournir des sources d'information (généralement des articles de presse ou de portails d'actualité) pour soutenir la véracité du cas. La validation du bloggeur consiste dans la vérification de la véracité des sources. Par exemple, le cas de l'immolation par le feu de Tang Fuzhen, une des nouvelles qui a fait le tour du globe, a été ajouté sur la carte le 16 octobre 2010 et il est documenté par un lien vers le site web Baidu, le principal moteur de recherche chinois (http://baike.baidu.com/view/1315584.html?fromTaglist) ${ }^{6}$.

Carte 1. La version non-vérifiée de la carte du sang de l'immobilier

\footnotetext{
${ }^{4}$ Interview - Arte Journal, http://www.arte.tv/fr/nav/3604810,CmC=3604832.html (consulté le 18 août 2011)

${ }^{5} \mathrm{~A}$ notre avis, la différence entre le nombre de cas déclarés et ceux vérifiés ne s'explique pas particulièrement par des déclarations sujettes à caution mais plutôt par le manque de temps du bloggeur qui n'arrive pas à vérifier tous les cas.

${ }^{6}$ Source : Alexa.com, 19 novembre 2011, http://www.alexa.com/siteinfo/baidu.com
} 


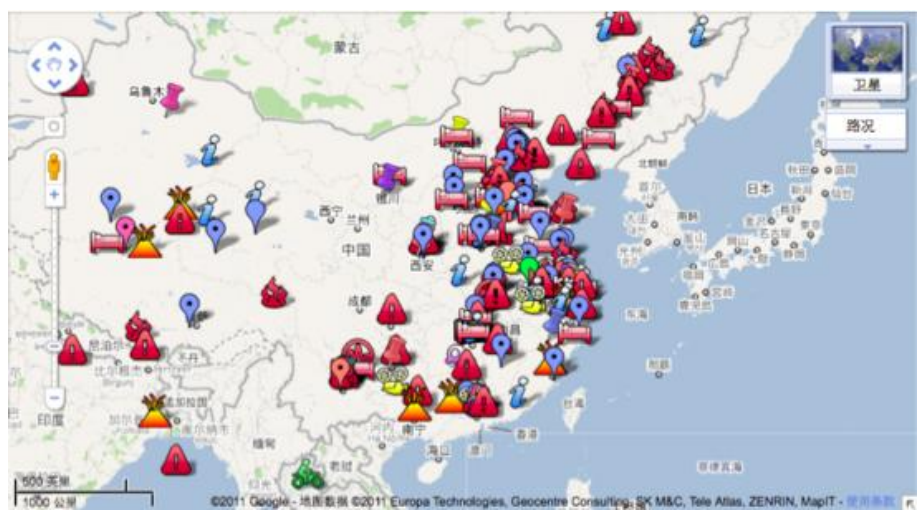

Carte 2. La version vérifiée de la carte du sang de l'immobilier

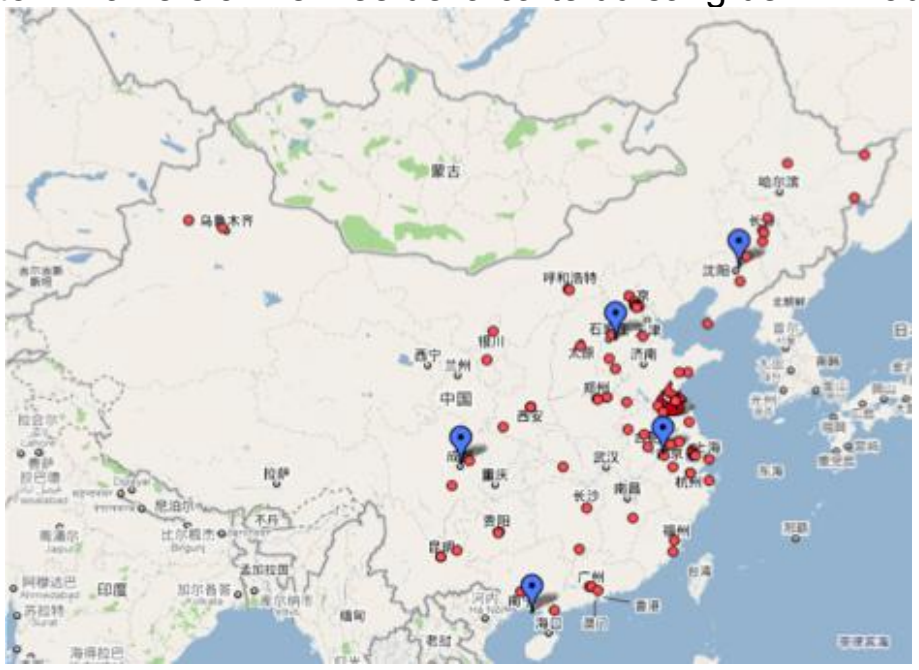

L'utilisateur peut zoomer sur la carte et y trouver la localisation exacte de l'incident, une petite icône symbolise aussi la nature de l'événement : un lit d'hôpital pour la mort d'un homme, une flamme pour une immolation par le feu ou encore un volcan pour une manifestation d'importance. Le point d'interrogation indique quant à lui que l'information n'a pas encore été vérifiée. Une étude statistique basée sur la carte vérifiée par le bloggeur révèle que $31 \%$ des cas concernent une manifestation, $23 \%$ entrainent au moins un mort ou encore $15 \%$ une immolation.

Figure 1. La nature des violences

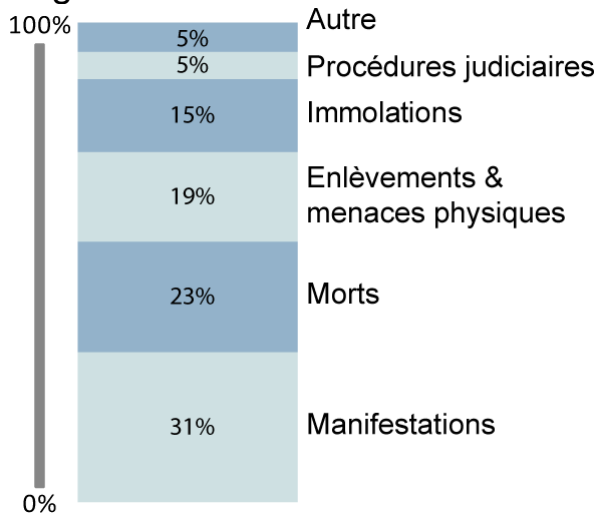


L'analyse statistique des déclarations met en avant comme causes des violences des indemnisations trop faibles ou encore des démolitions sans autorisations. Les victimes sont le plus souvent issues des milieux populaires et habitent les espaces centraux des villes qui connaissent d'importants projets urbains de modernisation et sont engagés dans des cycles de spéculation.

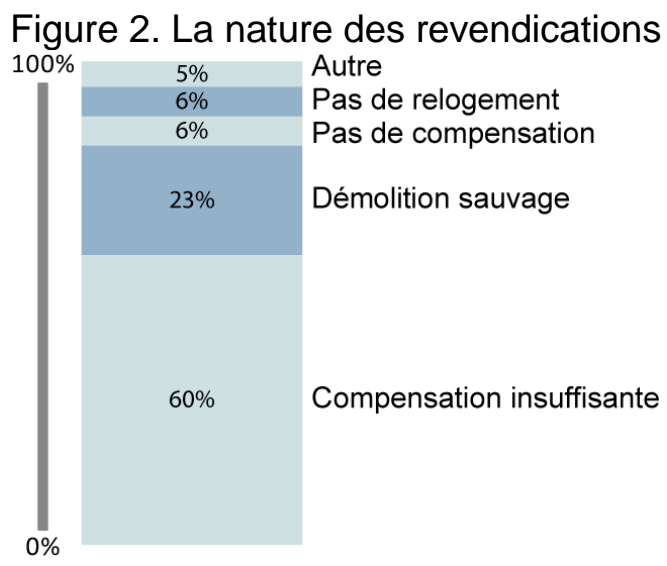

Figure 3. Le statut social des victimes

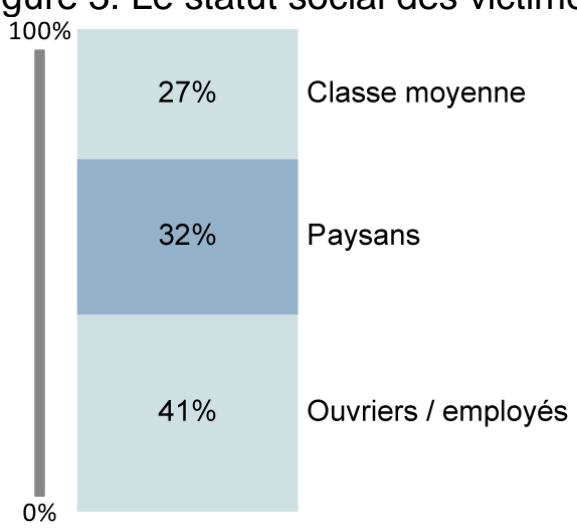

Figure 4. L'espace des événements

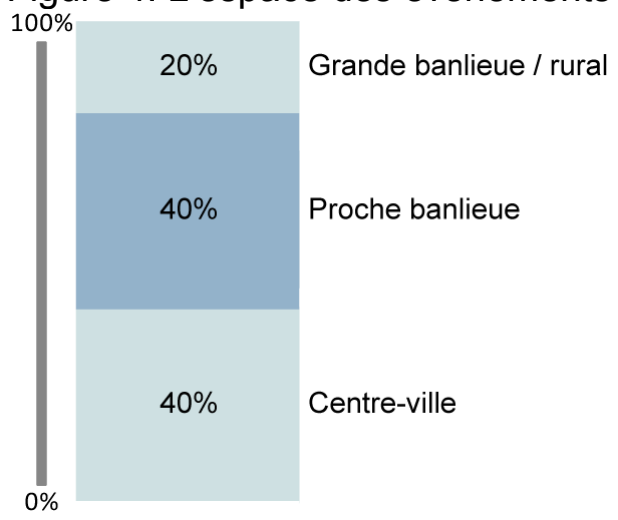

En étudiant dans le détail les pages web mentionnées comme source d'information des cas vérifiés, il émerge que les 85 cas de la carte vérifiée sont «prouvés » par 116 sources. Globalement, ces pages web proviennent à plus de $70 \%$ de portails d'actualité, de $12 \%$ de portails de communauté et seulement à $8 \%$ de journaux. Si quelquefois les 
contributeurs se servent des journaux comme le Weekend $d u S u d^{7}$, un des journaux les plus populaires de Chine car assez libéral, ou de l'agence de presse Chine Nouvelle ${ }^{8}$, la majorité des cas sont corrélés à d'autres types de source, notamment des sites web spécialisés (http://www.5law.cn qui fournit des conseils juridiques et http://news.cbi360.com qui est spécialisé dans le domaine de l'architecture) ou les principaux portails chinois, comme qq.com, sohu.com et $163 . c o m$, qui publient tous les types d'actualité. De plus, parmi les sources, un site web est particulièrement intéressant, il s'agit du portail boxun.com (http://news.boxun.com) qui est défini par Wikipedia comme étant une source alternative d'actualités en Chine $^{9}$.

Toutes ces données nous démontrent le poids et la largeur du phénomène des violences liées à l'immobilier. Si ces faits ne sont pas documentés par des statistiques officielles, ils n'obtiennent pas l'attention des autorités locales et nationales, ainsi le Web 2.0 leur offre une nouvelle importance et visibilité. Dans ce sens, la "carte du sang » permet surtout de rassembler des informations qui étaient dispersées. Grâce à l'initiative d'un bloggeur entreprenant et à l'action d'utilisateurs qui entendent profiter de ce nouveau terrain d'expression qui leur est offert par l'Internet, les abus reportés par les différents sites web locaux sont assemblés sur une plateforme unique. Par conséquent, la carte du sang donne une vision d'ensemble à niveau national d'un problème qui touche des gens et des contextes urbains disséminés sur l'étendue du territoire chinois.

\section{Le poids des réseaux médiatiques pour la diffusion de l'information}

La « carte du sang » a été publiée par le bloggeur le 8 octobre $2010^{10}$. À la fin décembre 2010, lorsque l'on tapait sur Google «Bloody map », on obtenait plus de 1,64 millions de résultats. L'information a circulé en Chine mais aussi au-delà, la France et les USA apparaissent comme les principaux foyers de diffusion.

Grâce à l'emploi de nouvelles méthodes numériques (Rogers, 2009) ${ }^{11}$, il a été possible de reconstruire la diffusion de l'information de la carte du sang à un niveau international. En utilisant plusieurs outils de repérage de liens et d'images ${ }^{12}$ sur la Toile, nous avons pu retrouver de nombreuses pages web qui ont parlé de la carte du sang. Nous avons identifié 139 sites mentionnant la carte et nous les avons analysé par type, par pays et

\footnotetext{
${ }_{8}^{7}$ http://www.infzm.com/enews/nfrwzk (consulté le 20 novembre 2011)

8 http://www.xinhuanet.com (consulté le 20 novembre 2011)

9 http://en.wikipedia.org/wiki/Boxun.com (consulté le 18 août 2011)

${ }_{10}^{10}$ Initialement sur le site chinois http://t.sina.com.cn/bloodymap puis à la suite d'une mesure de censure sur le site http://maps.google.com $/ \mathrm{maps} / \mathrm{ms}$ ?ie=UTF8\&msa=0\&msid=212261269395391329707.0004921f02f43f6c4f07e\&\|ll=35.3173 $66,111.357422 \& \mathrm{spn}=16.751302,43.286133 \& \mathrm{z}=5$ (consulté le 20 novembre 2011)

${ }^{11}$ Les méthodes qu'on regroupe sous cette étiquette sont très hétérogènes et encore peu intégrées. Cependant, elles ont en commun de se baser sur les traces numériques comme source d'information pour l'étude des phénomènes sociaux (Venturini \& Latour, 2010).

${ }^{12}$ Dans le détail, pour repérer les liens vers des pages qui parlent de la carte du sang nous avons employé deux types d'outil. Premièrement, nous avons employé des moteurs de recherche capables d'identifier des pages incluant un lien particulier, notamment la recherche avancée de Google dans plusieurs versions nationales (par exemple « Recherche de pages avec un lien vers la page suivante » à la page http://www.google.fr/advanced_search), le moteur de recherche SEO open site explorer (http://www.opensiteexplorer.org/) et Yahoo link explorer (http://siteexplorer.search.yahoo.com/). Avec ces outils nous avons cherché toute mention de la première adresse Internet du blog

(http://t.sina.com.cn/bloodymap) où a été publié initialement la carte, même si aujourd'hui ce site n'est plus accessible. Deuxièmement, nous avons employé des moteurs de recherche d'images capables d'identifier des pages incluant une image similaire ou identique à une image donnée, notamment Tineye (www.tineye.com) et la recherche par image de Google (http://www.google.fr/imghp?hl=fr\&tab=wi). Nous avons cherché toute occurrence de l'image de la carte (par exemple http://observers.france24.com/fr/files/imagecache/observers_520_320/oa/oa_image/carte2.jpg) et de la photo de Tang Fuzhen immolée par le feu (voir http://www.flickr.com/photos/29582880@N05/4190114970/), cas de violence souvent mentionné lors de la présentation de la carte dans les médias internationaux.
} 
par date de publication.

En ce qui concerne le type, la moitié des sites ont été classés en tant que site communautaire, c'est-à-dire blog, forum, wiki ou site de micro-blogging. L'autre moitié est principalement constituée par des portails d'actualités généralistes ou d'un thème spécifique, notamment liés à l'immobilier et à l'architecture (voir Figure 5). En ce qui concerne la distribution géographique, les sites sont repartis dans 19 pays, dont $40 \%$ provenant de Chine. Les sites web internationaux, qui ne sont pas localisables dans un seul pays (voir par exemple Globalvoice.com), sont également une partie importante du corpus. Puis la France (11\%), les Etats-Unis (8\%), I'Italie et l'Espagne (6\%) couvrent la plupart des sites web restants (voir Carte 3). Pour expliquer la distribution géographique, il est important de noter que GlobalVoices ${ }^{13}$, le plus important réseau international de bloggeurs et journalistes citoyens, a publié la nouvelle de la carte en anglais, français, coréen, bengali et espagnol. En comparant la catégorisation par type et par pays, nous avons remarqué un fait intéressant : dans les divers pays, la répartition par type est presque homogène, c'est-à-dire que la moitié des pages parlant de la carte dans chaque pays appartiennent à des sites web communautaires. Nous pouvons faire l'hypothèse qu'en Chine les informations concernant la carte ont été transmise surtout à travers le Web 2.0 pour éviter la censure alors que dans les autres pays ce sont surtout les médias traditionnels qui ont permis la circulation de l'information. Ces données nous montrent aussi que nous ne pouvons pas repérer une organisation de l'information spécifique aux divers pays, mais plutôt que, dans chaque contexte, les sites communautaires, les portails d'actualités et les journaux ont contribué à faire circuler l'actualité.

Enfin, nous avons analysé les sites en fonction de la date de publication des actualités concernant la carte du sang. Cela nous a permis de reconstruire dans le détail la diffusion de l'information à travers la planète entre le 8 octobre 2010, date de la publication de la carte, et le 14 Mars 2011, date de la dernière actualité repérée (voir figure 6). Après la publication sur le blog de «Bloody map », le 10 octobre l'information commence à circuler sur Twitter. Le 21 octobre le journal citoyen Boxun.com publie la nouvelle et seulement après (le 22) l'agence Chine Nouvelle (Xinhuanet) ainsi que l'Agence France Presse y consacrent des dépêches. Une semaine plus tard, une dizaine de médias chinois et étrangers en avaient parlé. Au même moment, le blog "Bloody map » est bloqué et la recherche des mots-clés «Bloody map » dans les moteurs de recherche chinois devenait censurée ${ }^{14}$.

Figure 5. Les sites web reprenant l'information de la carte du sang. Distribution par type et par pays.

\footnotetext{
${ }_{13}^{13}$ http://globalvoicesonline.org/ (consulté le 20 novembre 2011)

14 http://prix2011.aec.at/winner/1567/ (consulté le 20 novembre 2011)
} 


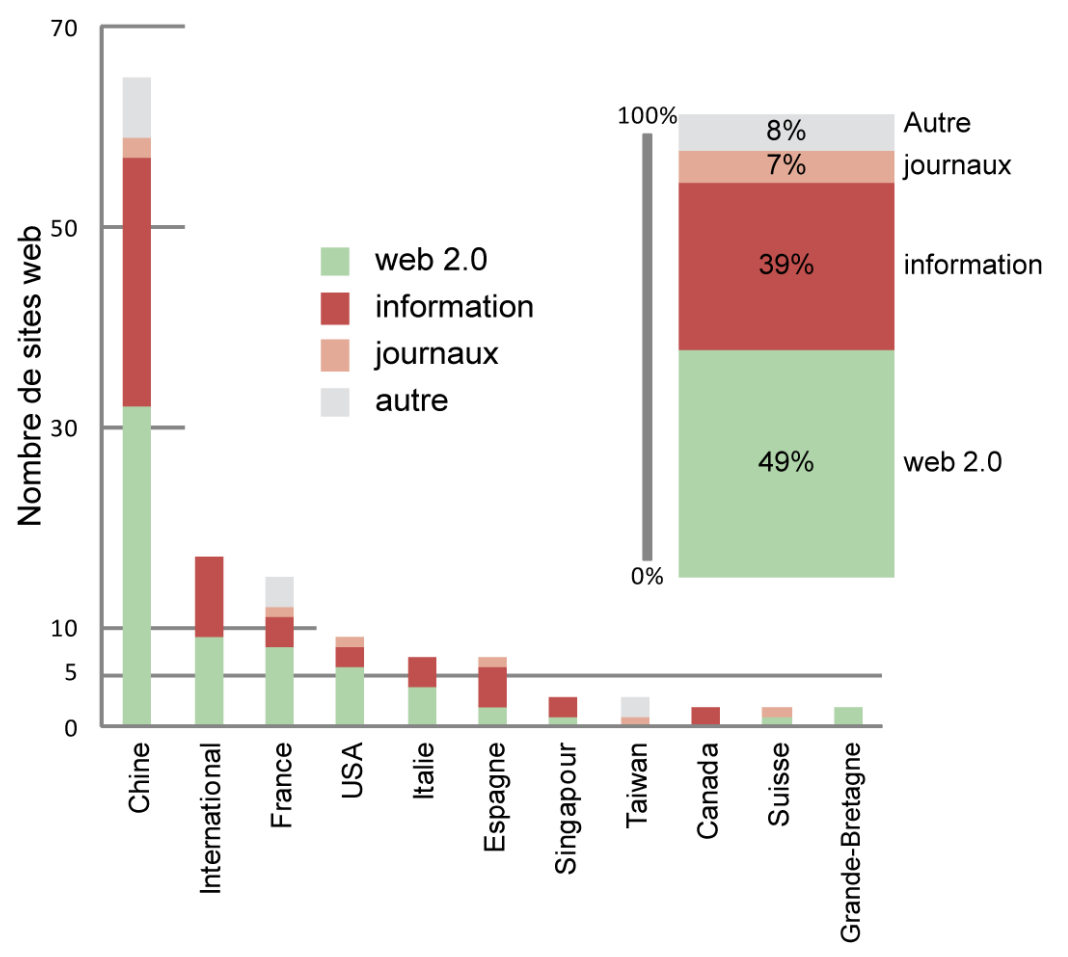

Carte 3. Les sites web reprenant l'information de la carte du sang

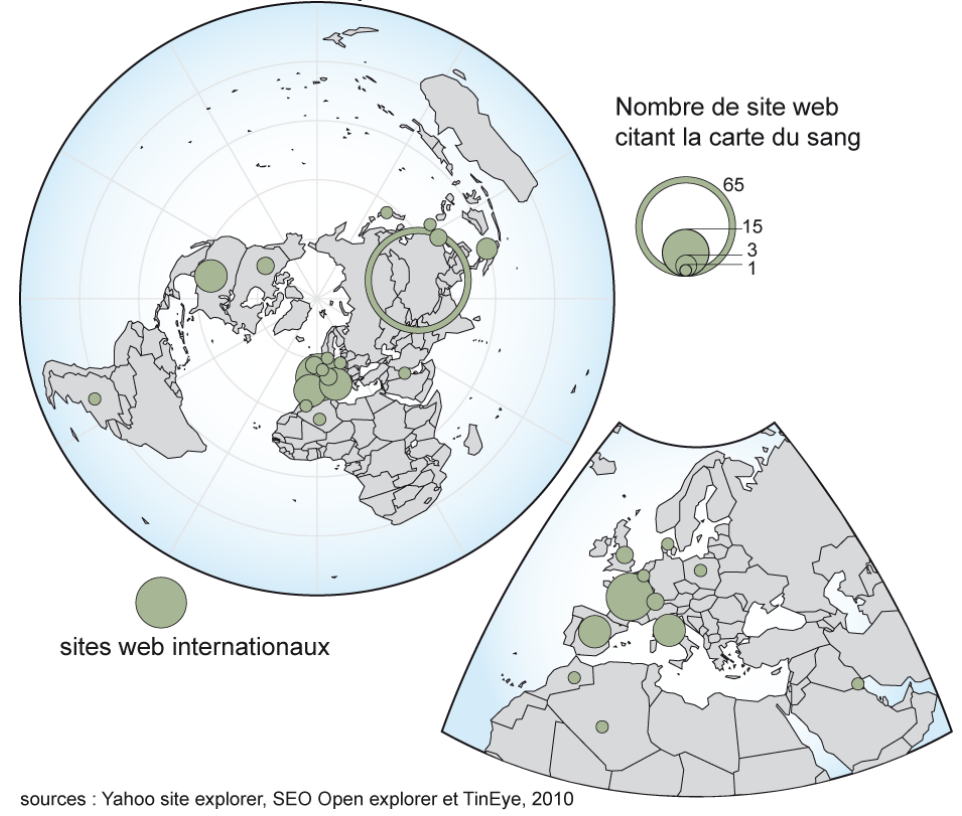

Figure 6. Les sites web reprenant l'information de la carte du sang. Distribution par date de publication. 


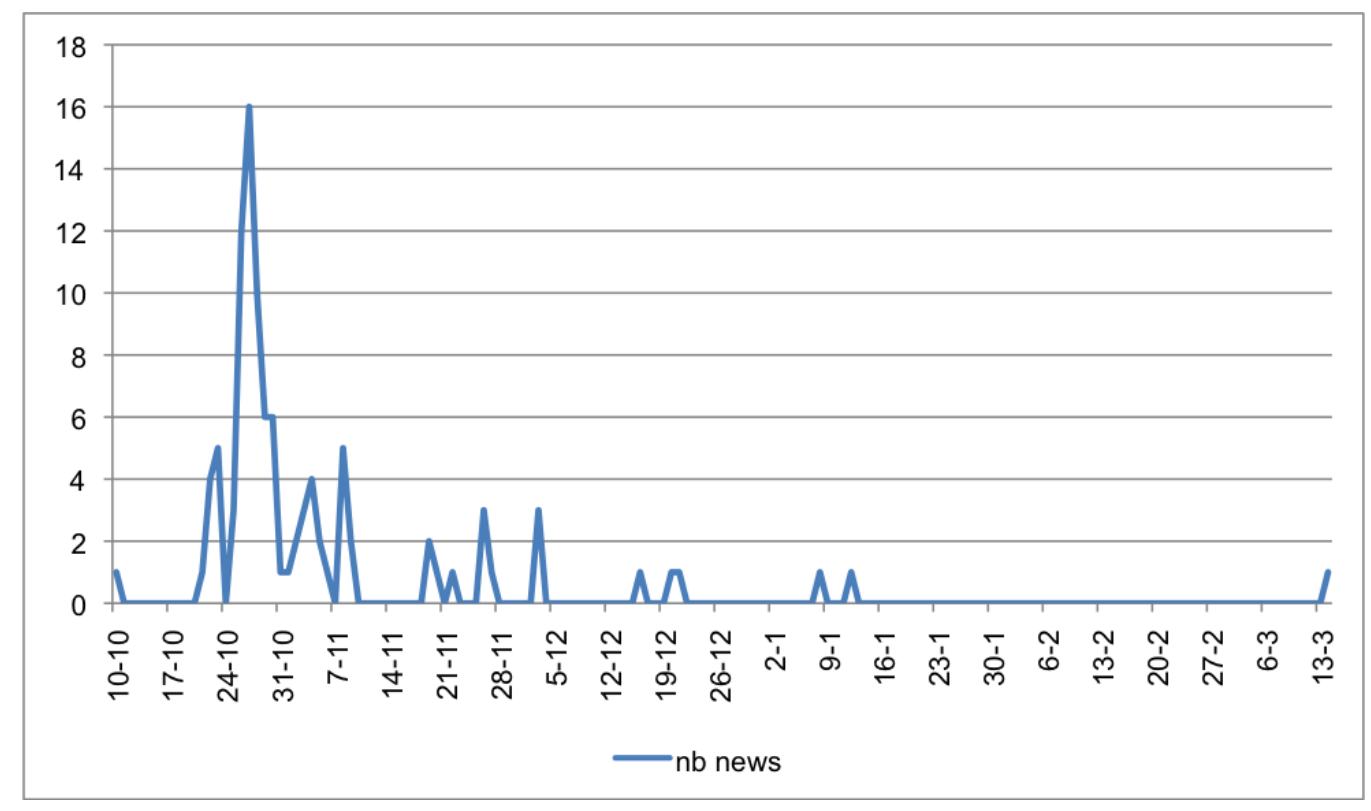

\section{Le Web 2.0, nouvel horizon de l'activisme urbain}

\section{De l'émotion au débat public}

Ces dernières années, l'espace public numérique chinois est devenu de plus en plus important. C'est en 2003 que l'Internet devient un outil majeur de dénonciation des abus de pouvoir et de la corruption. Pour la première fois, l'affaire Sun Zhigang révèle les capacités du Web à peser sur le débat public. Cet employé, migrant d'origine rurale qui venait d'obtenir son diplôme, est arrêté par des policiers alors qu'il n'est pas capable de présenter son permis de séjour ${ }^{15}$. II mourra lors de sa garde à vue suite aux violences des autorités, créant de ce fait une vive émotion dans l'opinion publique.

Au printemps 2007, l'événement majeur concerne une « maison clou $^{16}$ » de Chongqing. Seule rescapée d'un projet de promotion immobilière, elle est devenue mondialement célèbre grâce au bloggeur Zola ${ }^{17}$. Depuis 2004, Zhou Shuguang parcourt la Chine et rend compte dans son blog de différentes injustices. Lorsqu'il publie l'histoire de la maison de Chongqing, l'information est reprise dans toute la Chine mais aussi dans les principaux médias occidentaux, en commençant par la une du New York Times ce qui va permettre à l'information d'être reprise dans le monde entier. Zola accède de ce fait à la célébrité et il est contraint de faire héberger son blog en dehors de la Chine et de sécuriser son accès afin de déjouer les mécanismes de la censure. Avec cette médiatisation, le couple de propriétaires de Chongqing est devenu un symbole des luttes urbaines chinoises et finalement il s'est vu proposer une meilleure indemnisation par les autorités locales. Cette histoire a ainsi exposé, en Chine et bien au-delà, la question des

\footnotetext{
${ }^{15}$ Urbain ou rural, le hukou est un permis de résidence qui permet de contrôler l'exode rural. Le titulaire d'un hukou rural n'a pas les mêmes droits en matière d'éducation, de santé et de fiscalité que celui d'un hukou urbain.

${ }^{16}$ Une maison clou (dīngzihù) est une maison que le propriétaire refuse de céder ou de quitter en dépit de son inclusion dans un projet immobilier conditionné par la libération de la totalité des terrains alentour. L'expression est un jeu de mot inventé par les promoteurs immobiliers chinois pour faire référence à un clou en partie enfoncé dans du bois et qui ne peut disparaître qu'à coups de marteau.

${ }^{17}$ Le pseudonyme vient du footballeur italien Gianfranco Zola, Zhou Shuguang a découvert Émile Zola plus tardivement mais depuis il revendique aussi cette référence.
} 
expulsions d'habitants modestes pour laisser place à de nouveaux complexes immobiliers souvent réalisés dans une logique de spéculation immobilière.

Plus récemment, en novembre 2009, l'immolation par le feu de Tang Fuzhen suscita une vive émotion. Après une longue opposition au projet de démolition de sa maison dans la périphérie de Chengdu, cette femme de 47 ans s'est couverte à trois reprises d'essence sur le toit de sa maison alors que les démolisseurs pénétraient dans la demeure et rouaient de coups sa sœur et son mari. Elle mourut à l'hôpital, deux semaines plus tard, après d'atroces souffrances. Une vidéo de l'immolation réalisée à partir d'un téléphone sera largement diffusée sur le Web et finira même dans un reportage sur CCTV. Cet événement a touché l'opinion publique en la sensibilisant un peu plus sur les dérives des expulsions forcées liées à l'urbanisation galopante du pays.

En 2007 à Xiamen et en 2011 à Dalian, I'Internet chinois s'est mobilisé contre les risques industriels liés au paraxylène, un produit chimique toxique utilisé pour faire du polyester. Le 8 août 2011, des appels à la «balade » sont lancés en ligne (c'est à dire un appel détourné à la manifestation) et conduisent au rassemblement de plus de 10 000 personnes. Ils réclament le départ des installations industrielles car ils mettent en doute la capacité de résistance de celles-ci en cas de typhon (qui sont assez fréquents dans cette région). Quelques heures après le début de la manifestation le responsable local du parti communiste et le maire annoncent que l'usine sera délocalisée. Les protestations se poursuivent et les autorités finissent par annoncer l'arrêt immédiat de la production. Les événements de Xiamen et Dalian illustrent ainsi une mobilisation sociale en faveur des questions d'aménagement. Toutefois, l'objet des mobilisations s'élargie, les revendications ne sont plus uniquement matérialistes, par exemple autour des questions du logement, mais tendent à devenir post-matérialistes en intégrant les questions environnementales.

\section{Du réseau au débat public numérique}

Michel Offerlé (1996), politiste français, distingue trois grandes catégories de ressources mobilisables par les mouvements sociaux. Tout d'abord le nombre c'est-à-dire la capacité à mobiliser des effectifs importants. Ensuite l'expertise, dépendant des compétences des membres du groupe et/ou leur capacité à mobiliser des personnalités compétentes censées permettre la persuasion. Enfin, le recours au scandale pour dénoncer une situation et sensibiliser l'opinion publique. En plus du registre du scandale comme dans les médias traditionnels et les blogs, la dimension participative de la carte du sang de l'immobilier permet de s'appuyer sur les registres du nombre et de l'expertise pour se pérenniser. En théorie cette carte pourrait devenir un véritable outil de suivi et d'évaluation des politiques urbaines du gouvernement et par ailleurs une référence pour effectuer des choix éthiques de localisation résidentielle.

Alors que l'Internet semble accueillir plus facilement l'expression des différents mouvements sociaux urbains, les nouveaux outils du Web 2.0, comme les blogs et les réseaux sociaux, notamment les plateformes de micro-blogging qui sont moins soumises aux mécanismes de la censure, se transforment en forums de débat public offrant ainsi un espace public virtuel. Les plateformes en ligne permettent d'une part de contourner, en partie, les contrôles policiers rencontrés dans l'espace public physique. D'autre part, I'Internet met en relation les citoyens, rassemble des informations, fait circuler des mots d'ordre. Cette contestation 2.0 permet de rassembler un nombre important de citoyens 
en un temps plus court, de donner plus d'importance aux scandales et finalement de révéler des capacités inédites d'expertise qui apparaissent grâce à des processus participatifs. L'Internet révèle alors des mouvements sociaux urbains à l'organisation moins hiérarchique et collective laissant place à des formes d'engagement plus individuelles où la dimension réticulaire offre de nouveaux horizons en permettant l'apparition d'une intelligence collective.

\section{Conclusion : le cyber-activisme pour changer le cours des politiques publiques?}

Au-delà des scènes de conflit qui mobilisent ces cyber-citoyens, leurs revendications constituent une dénonciation des pratiques actuelles de l'aménagement urbain et des processus de fabrication de la ville. En élargissant l'espace du débat public, ils posent la question d'un tournant collaboratif en matière d'urbanisme. L'Internet et les réseaux sociaux permettent de façonner une opinion publique, de soutenir l'émergence d'une société civile. II reste à savoir si ces mouvements sociaux urbains permettront l'émergence de formes alternatives de leadership et rebondiront dans le territoire traditionnel du débat public afin d'influencer véritablement le cours des agendas politiques urbains. L'avenir dira si ces nouveaux territoires du débat public sont porteurs d'une nouvelle pratique de l'urbanisme, plus collaborative, plus durable ou encore plus inclusive.

\section{Bibliographie}

Arsène S. (2008), "Les discussions en ligne en Chine », in Perspectives chinoises, $\mathrm{n}$ ${ }^{\circ} 2008 / 2$, p. 88-99.

Centre on Housing Rights and Evictions (2008), One world, whose dream? Housing rights violations and the Beijing Olympic games

Douay N. (2011), «Urbanisme et cyber-citoyens chinois, la contestation 2.0 s'organise » in Perspectives chinoises, Hong Kong, Centre d'études français sur la Chine contemporaine, $\mathrm{n}^{\circ} 2011 / 1$, p. $86-88$

Douay N., Severo M. \& Giraud T. (2011), Les nouveaux territoires du débat public, le cas de la carte du sang de l'immobilier chinois, Colloque fondateur du CIST «Fonder les sciences du territoire », Paris, 23-25 novembre

Haski P. (2008), Internet et la Chine, Paris, Seuil

Hermès n55 (2010), « Société civile et Internet en Chine et Asie orientale »

Kurt Herold D. \& Marolt P. (dirs) (2011), Online Society in China: Creating, Celebrating, and Instrumentalising the Online Carnival, London, Routledge

Human Right Watch (2003), Demolished: Forced evictions and tenant's right movement in China, mars 2004, vol.16, $\mathrm{n}^{\circ} 4$

Li J. \& Wang Y. (2011), La cyber-mobilisation face au développement immobilier chinois : le cas de la carte du sang, mémoire de master de Géographie, (dir. Douay N.), Université Paris-Diderot 
Offerlé M. (1996), Sociologie des groupes d'intérêt, Paris, Montchrestien

Rogers, R., 2009, The End of the Virtual: Digital Methods, Amsterdam, Amsterdam University Press.

Severo M., Giraud T. \& Douay N. (2011), Citizen protest in the online networks: the case of the China's bloody map, 7th UK Social Networks Conference, University of Greenwich, Londres, 7-9 juillet

Tai Z. (2006), The Internet in China: Cyberspace and Civil Society, NYC, Routledge

Yang G. (2009), The Power of the Internet in China: Citizen Activism Online, NYC, Columbia University Press

Venturini, Tommaso, \& Latour, Bruno (2010), The Social Fabric: Digital Traces and Quali-quantitative Methods, Proceedings of Future En Seine 